\title{
Editorial \\ Distance Learning: Pitfalls and Possibilities
}

\author{
Anna Felnhofer ${ }^{1} \&$ Oswald D. Kothgassner ${ }^{2}$ \\ 1 Department of Pediatrics and Adolescent Medicine, Medical University of Vienna, Austria \\ ${ }^{2}$ Department of Child and Adolescent Psychiatry, Medical University of Vienna, Austria
}

DOI 10.24989/dp.v2i2.2020

The manifold COVID-19 measures have accelerated digitalization, particularly with regards to staying connected with others and continuing one's education in the face of lockdowns and quarantine. Even before the crisis, digitalization has begun to fundamentally change how we teach and learn (Pettersson, 2021). Yet, the introduction of emergency distance learning in early 2020 has led to an unparalleled pervasiveness of video conferencing-platforms in educational settings. While this certainly comes with multiple challenges for both teachers and students, it may also entail promising opportunities for enhancing traditional educational methods. The current editorial will - cursory and by no means exhaustively - pick up on several open research questions and ongoing developments.

\section{Potential pitfalls}

Amongst others, it has been suggested that videoconferencing tools may negatively impact the ability to effectively empathize with others. Studies exist for online psychotherapy (Grondin et al., 2019), but findings may also be transferred to the educational setting. Specific characteristics of the medium are thought to alter the way we socially interact online, such as reduced nonverbal cues (mimics, gestures), a decreased synchronicity and immediacy of feedback, problems with transmission quality, and a transformed content of communication (e.g., face-only instead of whole-body-view, altered eye contact). The increased demands on attentional resources arising from these circumstances may hinder attention and lead to tiredness, all of which has been coined as "Zoom fatigue" (Wiederhold, 2020).

Another challenge is brought on by the fact that - in most cases - we see ourselves on screen when talking to others. Some studies have found this to be distracting and to divert attentional resources from the task and, which, in turn, diminishes performance (Payne et al., 2020). Also, seeing oneself deviates from our common experiences in face-to-face communication and - given the increased self-focus - may be particularly challenging for those who are socially anxious (Clark \& Wells, 1995).

At the extreme, videoconferencing platforms may also facilitate cyberbullying. Several instances of teacher and peer bully- ing have been described since the implementation of COVID-19 associated measures (e.g., Ambrožová, \& Kaliba, 2021). Factors which may increase the risk of cyberbullying include protected anonymity, social distance, and ever-present availability; particularly, the lack of eye contact has been hypothesized to act as a facilitator for cyberbullying (Grondin et al., 2019).

\section{Table of Contents}

\section{Editorial}

3 Discrepancies Between Self-Reports and Behavior: Fear of Missing Out (FoMO), Self-Reported Problematic Smartphone Use Severity, and Objectively Measured Smartphone Use Dmitri Rozgonjuk, Jon D. Elhai, Onur Sapci \& Christian Montag

11 Attachment Insecurity and Social Media Fear of Missing Out: The Mediating Role of Intolerance of Uncertainty Yitshak Alfasi

19 Emergency Distance Learning in Austria during COVID-19: Selected Findings and Implications Elisabeth Pelikan, Katharina Hager, Julia Holzer, Selma Korlat, Christiane Spiel, Barbara Schober \& Marko Lüftenegger

23 Art and Design Education in Times of COVID-19: Distance Learning and the Importance of Interaction and Empathy Ruth Mateus-Berr

27 Piloting of Virtual Patient-Based Online Self-Study Quizzes for Developing Undergraduate Medical Students' Clinical Reasoning Skills Alexandra Rogler, Sophie Freilinger, Peter Pokieser \& Michaela Wagner-Menghin 


\section{Potential possibilities}

Apart from these pitfalls, computer mediated communication may also be used to enhance teaching and learning. For instance, a recent analytical report (Flecha et al., 2020) found that particularly rich and dialogic online interactions may promote empathy in children. Based on the self-awareness theory (Duval \& Wicklund, 1972), seeing one's video may be regarded as an enhanced state of self-awareness. Self-awareness, in turn, triggers comparisons between one's current behavior and standards of normative behavior, and hence, may increase empathy (Miller et al., 2017).

Overall, enhancing medium richness seems to be the way to counteracting possible pitfalls of videoconferencing technology: For example, fully immersive virtual reality (VR) technology may overcome some restrictions of $2 \mathrm{D}$-video interactions by allowing for the inclusion of a broader range of nonverbal cues (i.e., body language, gestures) and for embodiment (i.e., being represented in VR via an avatar). In addition, rich media like 3D-VR have also been shown to enhance the experience of social presence. Social presence describes the sense of "being together" and is thought to facilitate digital social interactions (Biocca et al., 2001).

\section{Outlook}

Currently, online conferencing tools constitute a less than ideal but necessary tool that enables interaction even in the face of restrictions like those associated with the COVID-19 crisis. Research is presently picking up on key topics of computer mediated communication in the context of education, and the future will certainly bring an improvement in the implementation of these technologies. Open issues include the consideration of different age groups in research; for instance, children and adolescents are expected to adopt new technologies differently from older adults because of their early socialization with technologies and their divergent cognitive and emotional development. Furthermore, to ensure inclusion and participation, culture and gender sensitive studies are needed which focus on the individual needs and prerequisites (e.g., access to technologies) of different societal groups.

Anna Felnhofer and Oswald D. Kothgassner

Editors-in-Chief

\section{References}

Ambrožová, P., \& Kaliba, M. (2021). Teacher-shaming in the context of Czech distance learning due to COVID-19 pandemic. In Proceedings of EDULEARN21 Conference (Vol. 5, p. 6th), July.

Biocca, F., Harms, C., \& Gregg, J. (2001, May). The networked minds measure of social presence: Pilot test of the factor structure and concurrent validity. In 4th annual international workshop on presence, Philadelphia, PA (pp. 1-9).

Clark, D. M., \& Wells, A. (1995). A cognitive model of social phobia. In R. G. Heimberg, M. R. Liebowitz, D. A. Hope, \& F. R. Schneier (Eds.), Social phobia: Diagnosis, assessment, and treatment (pp. 69-93). The Guilford Press.

Duval, S., \& Wicklund, R. A. (1972). A theory of objective self awareness. New York: Academic.

Flecha, R., Pulido, C., Villarejo, B., Racionero, S., Redondo, G., \& Torras, E. (2020). Effects of the Use of Digital Technology on Children's Empathy and Attention Capacity. Analytical Report. European Commission.

Grondin, F., Lomanowska, A. M., \& Jackson, P. L. (2019). Empathy in computer-mediated interactions: A conceptual framework for research and clinical practice. Clinical Psychology: Science and Practice, 26(4), e12298.

Miller, M. K., Mandryk, R. L., Birk, M. V., Depping, A. E., \& Patel, T. (2017, May). Through the looking glass: The effects of feedback on self-awareness and conversational behaviour during video chat. In Proceedings of the $2017 \mathrm{CHI}$ conference on human factors in computing systems (pp. 5271-5283).

Payne, L., Flannery, H., Kambakara Gedara, C., Daniilidi, X., Hitchcock, M., Lambert, D., ... \& Christie, D. (2020). Business as usual? Psychological support at a distance. Clinical child psychology and psychiatry, 25(3), 672-686.

Pettersson, F. (2021). Understanding digitalization and educational change in school by means of activity theory and the levels of learning concept. Education and Information Technologies, 26(1), 187204.

Wiederhold, B. K. (2020). Connecting through technology during the coronavirus disease 2019 pandemic: Avoiding "Zoom Fatigue", Cyberpsychology, Behavior, and Social Networking, 23(7), 437-438.

\section{Conflict of interest}

The Editors-in-Chief declare no conflict of interest. 